\title{
INFLUENCE OF THE DAMPING EFFECT ON THE DYNAMIC RESPONSE OF A PLATE
}

\author{
ŁUKASZ BORKOWSKI \\ Lodz University of Technology, Department of Strength of Materials, Eódź, Poland \\ e-mail: lukasz.borkowski@p.lodz.pl
}

\begin{abstract}
The subject of the research is analysis of the influence of the damping effect on the dynamic response of a plate. During the tests, the areas of dynamic stability and instability for the plate with and without damping are compared. Besides, exact analysis of the nature of the solution by applying criteria such as phase portraits, Poincaré maps, FFT analysis, the largest Lyapunov exponents are carried out and found.

Key words: damping effect, dynamic stability, dynamic response, phase portraits, Poincaré maps
\end{abstract}

\section{Introduction}

The beginnings of studies concerning the dynamic stability of plates can be found in publications from the middle of the twentieth century. The first publication regarding dynamic stability of plates was presented by Zizicas (1952). In that paper, theoretical solutions for the joint supported plate with a time-dependent load were reported. Subsequent years of research led to creation of dynamic stability criteria which were divided into: geometric (Cooley and Tukey, 1965), energy (Raftoyiannis and Kounadis, 2000) and failure ones (Petry and Fahlbusch, 2000).

One of the major criterion was a Budiansky-Hutchinson criterion (Hutchinson and Budiansky, 1966) which concerned rods and cylindrical shells with an axial load. They analyzed the load in the form of a pulse of a finite and infinite duration. They proved that the loss of stability of dynamically loaded constructions occurs when small load increments cause a rapid increase of deflection. Budiansky was one of the authors of a similar criterion regarding cylindrical shells with a transverse load - the Budiansky-Roth criterion (Budiansky and Roth, 1962). This criterion was willingly used in research of other scientists who were involved in the similar topics (Shariyat, 2007; Kubiak, 2007; Zhang et al., 2004).

Another important criterion is the Petry-Fahlbusch criterion (Petry and Fahlbusch, 2000). The researchers said that the analysis of the stress state should determine the dynamic critical load for the construction with a stable post-critical equilibrium path. Based on such an analysis, it is possible to determine the load for which destruction of the structure takes place. According to Petry-Fahlbusch's theory, if the condition - the reduced stress is smaller or equal to the boundary stress - is fulfilled at any time and at any point of the studied structure, then a dynamic response of the construction under the pulse load is dynamically stable.

The next important criterion is the Volmir criterion (Volmir, 1972). He analyzed pulses of a finite duration: a rectangular pulse and an exponentially decreasing pulse, pulses of an infinite duration and a linearly increasing load. He studied pulses that caused both compression and shear. Using the Bubnov-Galerkin (Michlin and Smolnicki, 1970) and Runge-Kutta (Collatz, 2012; Fortuna et al., 2005) methods, he said that the loss of stability of pulse loaded plates occurs when the maximum deflection of the plates is equal to their thickness or half thickness. 
Ari-Gur and Simonetta (1977) proposed four criteria for the loss of stability. They described the critical load depending on the following parameters: the measured deflection in the middle of length and width of the plate and the intensity of load for plates fixed at all edges and loaded with a pulse of half-wave shaped (a pulse of finite duration). The first concerns the value of deflection and the intensity of the load pulse - if a slight increase in the load pulse intensity causes a significant increase in the value of deflection then dynamic buckling takes place. According to the second criterion - if a slight increase in the amplitude of the load pulse causes a decrease in the value of deflection then dynamic buckling happens. The next two criteria are failure criteria which are based on the response analysis of the loaded edge of a plate. According to the third criterion - if a small increase in the force pulse amplitude causes a sudden increase in the shortening value of the loaded edge of the plate then dynamic buckling occurs. According to the fourth criterion - if a small increase in the pulse intensity of displacement of the loaded edge causes a change in the reaction sign on the plate edge then dynamic buckling takes place.

The behavior of rod systems was analyzed by the finite element method by Kleiber et al. (1987). They formulated a quasi-bifurcation criterion of dynamic stability for a construction under a jump loaded (Heaviside pulse) by using properties of a tangent stiffness matrix in the point of bifurcation. According to this criterion, the structure loses stability and the deflection begins to grow boundlessly when the determinant of the tangent stiffness matrix is equal to zero and the absolute value of the smallest eigenvalue is greater than the absolute value of the nearest maximum reached by the smallest eigenvalue.

All the above criteria are widely used in the research of many scientists who deal with the analysis of dynamic stability (Bolotin 1972; Hsu and Forman, 1975; Kołakowski, 2007; Kołakowski and Kubiak, 2007; Kowal-Michalska, 2010; Kubiak et al., 2010; Mania and Kowal-Michalska, 2007; Moorthy et al., 1990; Wu and Shih, 2006).

However, the analysis of plate structures with application of dynamic criteria such as phase portraits, Poincaré maps, FFT analysis, the largest Lyapunov exponents is less used (Alijani et al., 2011a,b; Gilat and Aboudi, 2000; Touati and Cederbaum, 1995; Wang et al., 2010; Yeh and Lai, 2002; Yuda and Zhiqiang, 2011). Therefore, this paper presents the influence of the damping effect on the dynamic response of the plate using the above tools.

\section{Studied plate}

A square isotropic plate with dimensions $b=l=100 \mathrm{~mm}, h=1 \mathrm{~mm}$ and material constants $E=200 \mathrm{GPa}, \nu=0.3$ is analyzed (Fig. 1). The analyzed plate is simply supported on the all edges. The plate is loaded with a dynamic compressive load. The dynamic load means the load that has been introduced suddenly and lasts for an infinitely long time.

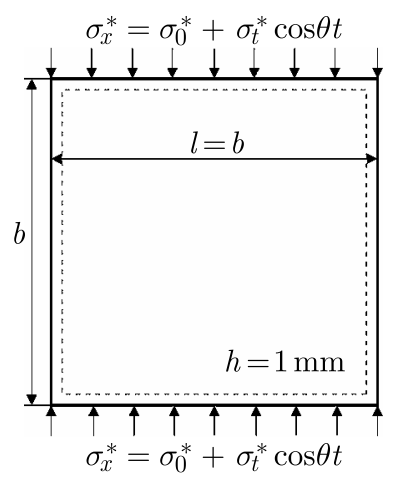

Fig. 1. Studied plate 


\subsection{The plate without damping}

According to the research by Volmir (1972), the above plate can be described by the following equation

$$
\ddot{\zeta}+\omega_{0}^{2}\left(1-\frac{\sigma_{x}^{*}}{\sigma_{c r}^{*}}\right) \zeta+\eta \zeta^{3}=0
$$

After transformations, the test plate without damping can be described using the equation

$$
\ddot{\zeta}+\Omega_{0}^{2}(1-k \cos \theta t) \zeta+\eta \zeta^{3}=0
$$

where

$$
k=\frac{\sigma_{t}^{*} / \sigma_{c r}^{*}}{1-\sigma_{0}^{*} / \sigma_{c r}^{*}} \quad \Omega_{0}^{2}=\omega_{0}^{2}\left(1-\frac{\sigma_{0}^{*}}{\sigma_{c r}^{*}}\right)
$$

and $\zeta$ is the deflection of the plate, $\omega_{0}$ - natural frequency, $\sigma_{c r}^{*}-$ critical stress, $\sigma_{0}^{*}-$ medium stress, $\sigma_{t}^{*}$-stress amplitude, $\eta$ - parameter whose value is dependent on the boundary conditions.

Transform now equation (2.2) into a dimensionless form

$$
\ddot{x}+a(1-k \cos \psi \tau) x+b x^{3}=0
$$

where

$$
a=1-\frac{\sigma_{0}^{*}}{\sigma_{c r}^{*}} \quad b=\frac{\eta}{\omega_{0}^{2}} \quad \ddot{x}=\frac{1}{\omega_{0}^{2}} \ddot{\zeta} \quad x=\zeta \quad x^{3}=\zeta^{3} \quad \psi=\frac{\theta}{\omega_{0}}
$$

and $\tau$ is the dimensionless time. For the studied plate supported on all edges, the values of parameters are: $\omega_{0}=3014.3 \mathrm{rad} / \mathrm{s}, \eta=0.23 \mathrm{rad} / \mathrm{s}^{2}, \sigma_{c r}^{*}=72.3 \mathrm{MPa}$. For the purpose of further numerical analysis, equation (2.3) is replaced by two first-order differential equations

$$
\dot{x}_{1}=x_{2} \quad \dot{x}_{2}=-a(1-k \cos \psi \tau) x_{1}-b x_{1}^{3}
$$

\subsection{The plate with damping}

Introducing damping into equation (2.2) and transforming into a dimensionless form, one obtains

$$
\ddot{x}+c \dot{x}+a(1-k \cos \psi \tau) x+b x^{3}=0
$$

where: $c=2 h / \omega_{0}$ - the dimensionless damping ratio, $h=0.02$ (Kołakowski and Teter, 2013), the other parameters are the same as for the plate without damping.

Writing equation (2.5) in the form of two first-order differential equations, we get

$$
\dot{x}_{1}=x_{2} \quad \dot{x}_{2}=-c x_{2}-a(1-k \cos \psi \tau) x_{1}-b x_{1}^{3}
$$

All studies are carried out for the following initial conditions: $x_{1}=0.01, x_{2}=0$.

\section{Numerical analysis of the plate}

Figure 2 shows the areas of dynamic stability and instability (circled areas) for the plate without (a) and with (b) the damping effect after earlier presentation of the full compliance of the results presented by Volmir (1972) and the results obtained with the dynamic tools for the plate without the damping effect (Borkowski, 2017). 
(a)

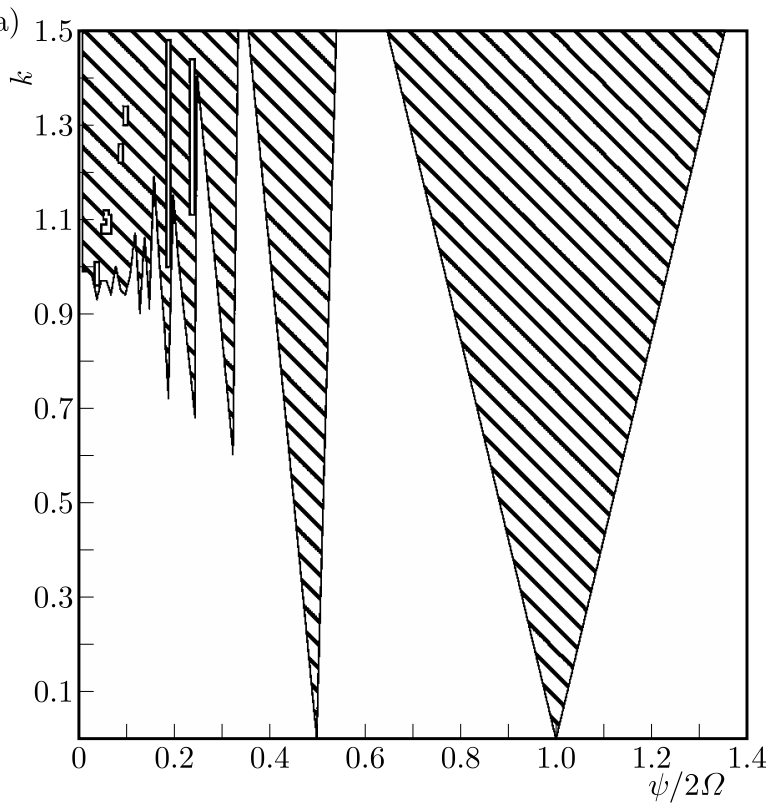

(b)

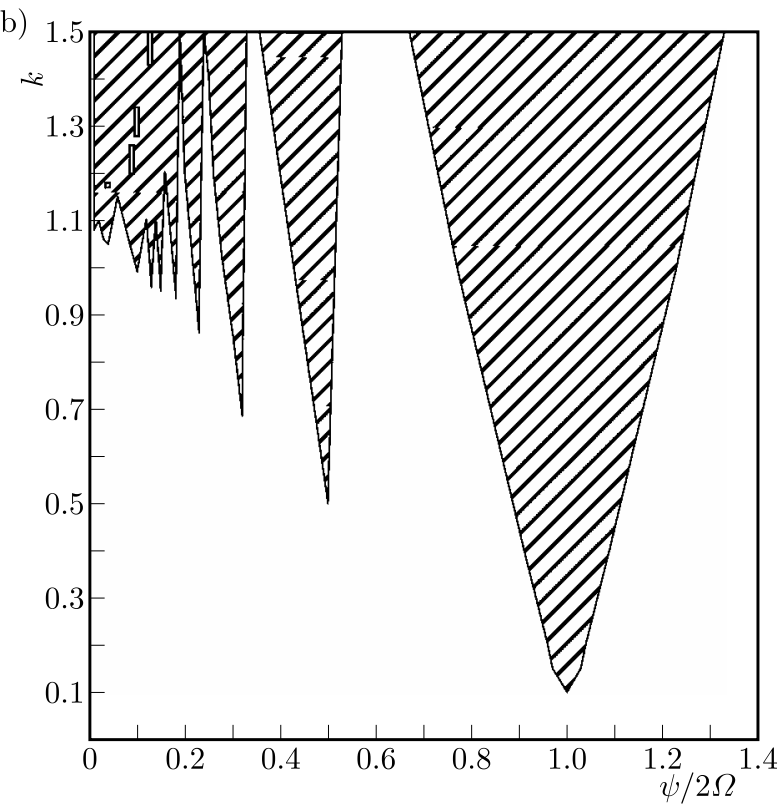

Fig. 2. Graphs of dynamic stability and instability areas for the plate without (a) and with (b) the damping effect

Both graphs in $k-\psi / 2 \Omega$ coordinates $\left(\psi=\theta / \omega_{0}, \Omega=\Omega_{0} / \omega_{0}\right)$ by changing values of the parameters $\sigma_{0}$ and $\sigma_{t}$ have been made. Calculations of the parameters $k$ and $\psi / 2 \Omega$ changing every 0.01 were executed. Figure 2 has been obtained by using the criteria of phase portraits, Poincaré maps and FFT analysis.

Analyzing both charts, it can be concluded that there are larger areas of dynamic instability for the plate without damping as against the plate with damping. In addition, small dynamic stability areas within the dynamic instability range in both cases are observed (Figs. 3a and 3b).
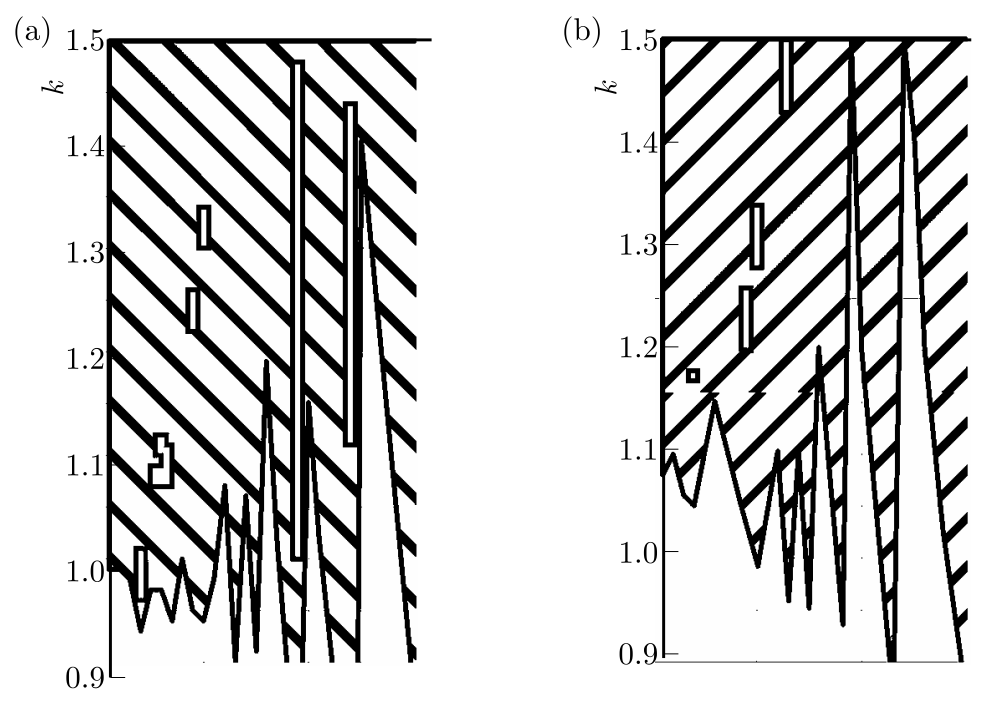

Fig. 3. Detailed graphs of dynamic stability and instability areas for the plate without (a) and with (b) the damping effect

For the plate without damping, the dynamic stability area is represented by a quasi-periodic solution. In the range of dynamic instability, both quasi-periodic as well as chaotic solutions can be specified. 
For the plate with damping, in the dynamic stability area the trajectory is heading to the critical point. In the range of dynamic instability, the periodic solutions as well as the series of period-doubling bifurcations, which lead to a chaotic response, are obtained.

Therefore, for the purpose of a more detailed analysis and presentation of the above solutions, the criterion of the largest Lyapunov exponents has been used.

Figure 4 shows the areas of the chaotic solution (gray areas) for the plate without (a) and with (b) damping. The dashed lines indicates the boundary for the dynamic stability/instability areas which corresponds to the circled part in Fig. 2. Comparing the two graphs, it can be clearly stated that the introduction of damping to the analyzed plate results in obtaining much smaller areas of dynamic instability with a chaotic solution. Figure 5 presents a magnification of Figs. 4a and 4b. Gray dots correspond to specific values and gray lines to ranges for the chaotic solution.
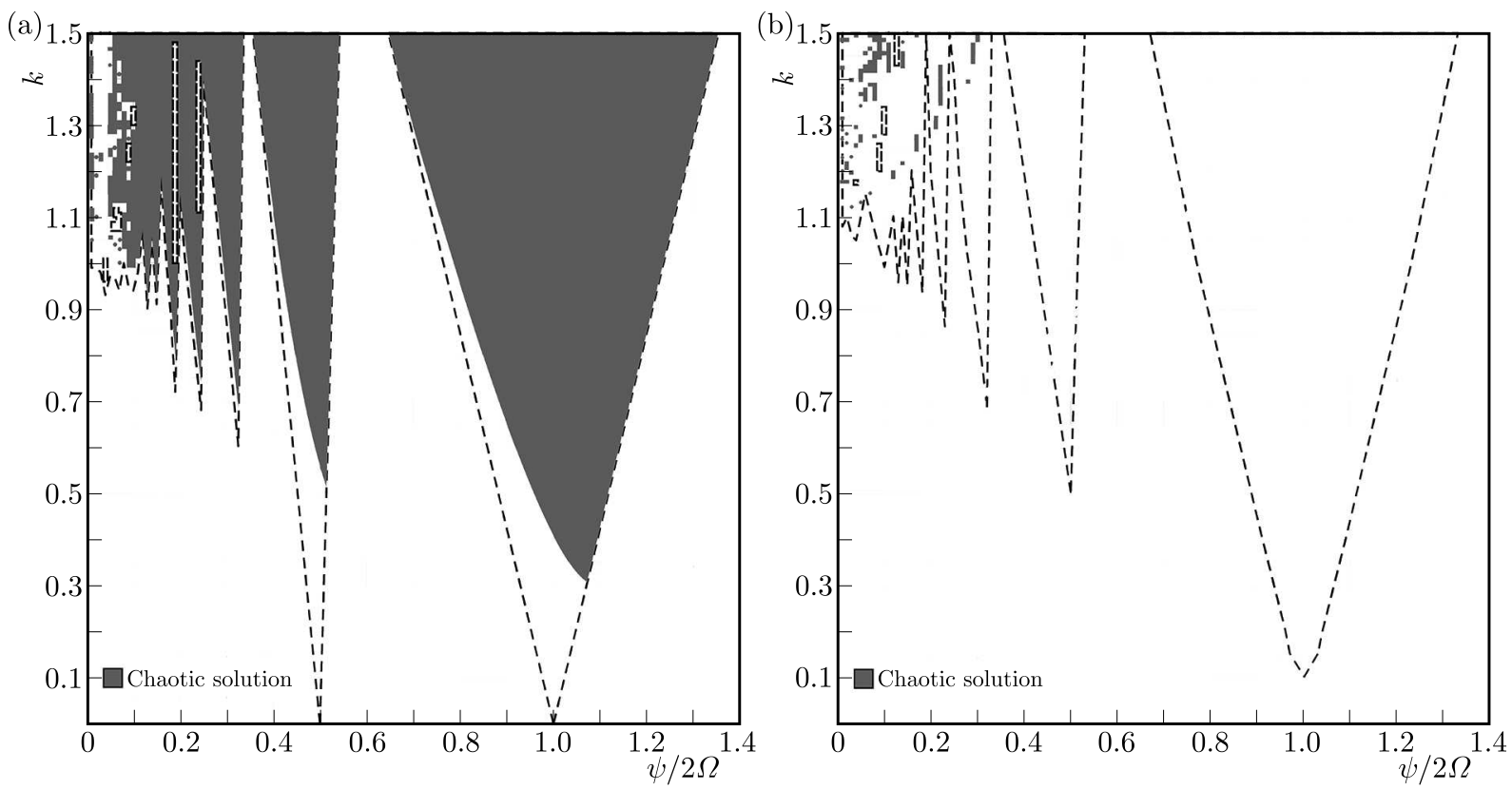

Fig. 4. Graphs of areas representing the chaotic solution for the plate without (a) and with (b) the damping effect

(a)

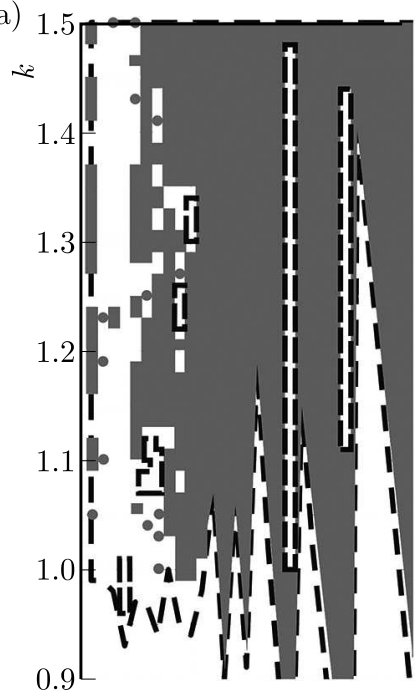

(b)

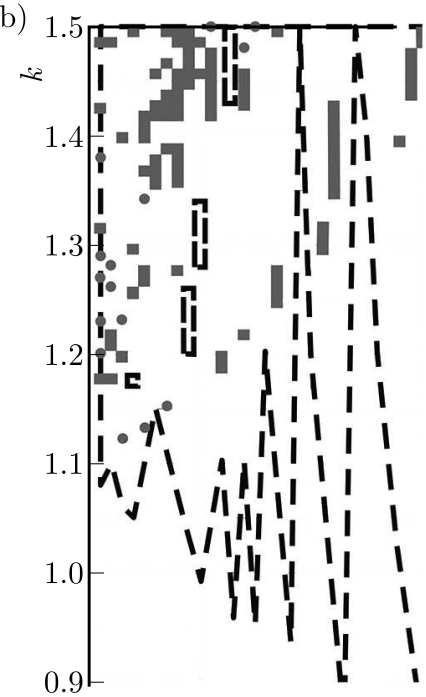

Fig. 5. The detailed graphs of areas representing the chaotic solution for the plate without (a) and with (b) the damping effect 
In order to present the solutions more clearly, three points from Fig. 2 for individual ranges have been selected. These points represent solutions from the area of dynamic stability $(k=0.50, \psi / 2 \Omega=0.30)$, from the area of dynamic instability with a periodic/quasi-periodic solution $-(k=0.25, \psi / 2 \Omega=1.00)$ and from the area of dynamic instability with a chaotic solution $-(k=1.50, \psi / 2 \Omega=0.30)$.

Analyzing the obtained results and using the criteria of phase portraits as well as Poincaré maps, it can be concluded that the loss of dynamic stability is associated with a sudden increase in the displacement $x_{1}$ and velocity $x_{2}$ (Figs. 6d, 6f, 6g, 6i) when compared to the dynamic stability areas (Figs. 6a, 6c). According to the research presented in (Bazant and Cedolin, 2010), the loss of stability is related to displacement of the phase trajectory into infinity. This is the case when the analysis time corresponds to the period of natural vibration of a construction. In order to use dynamic tools such as phase portraits or Poincaré maps, the presented research concerns the analysis duration many times greater than the period of natural vibration. For a long duration, the phase trajectory does not move into infinity. It achieves some limit values of the displacement $x_{1}$ and velocity $x_{2}$, the value of which depends on the parameter $k$. However, applying the criterion of phase portraits and analyzing the plate for both short and long analysis duration, the same results are obtained.

(a)

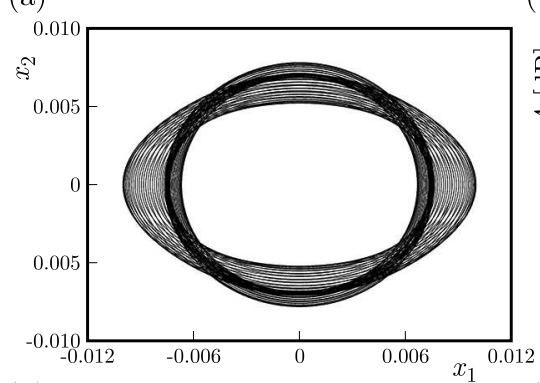

(d)

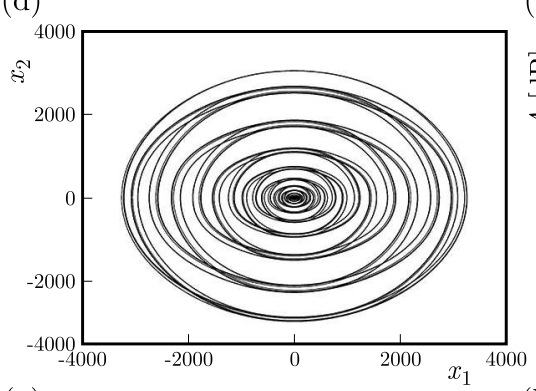

(g)

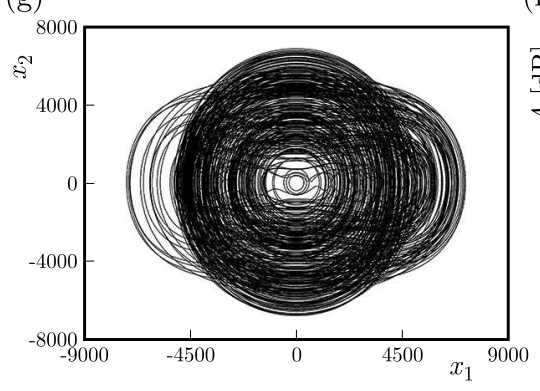

(b)

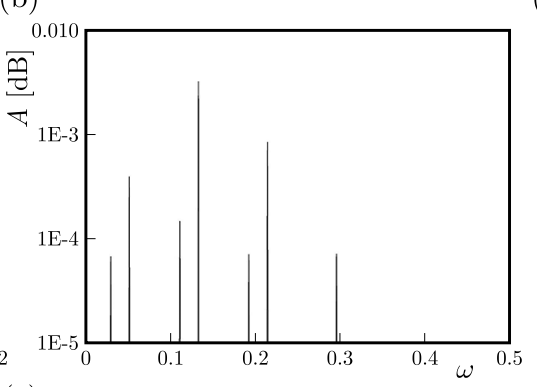

(e)

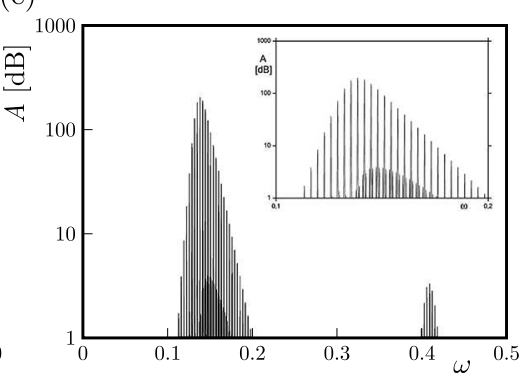

(h)

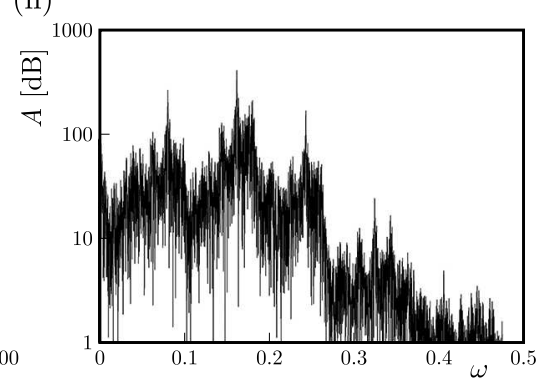

(c)

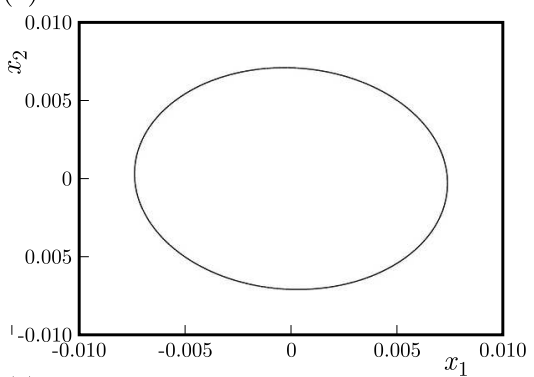

(f)

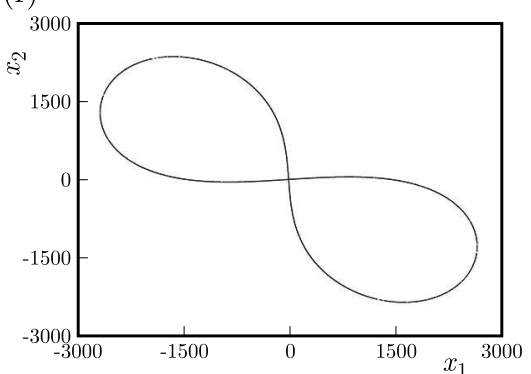

(i)

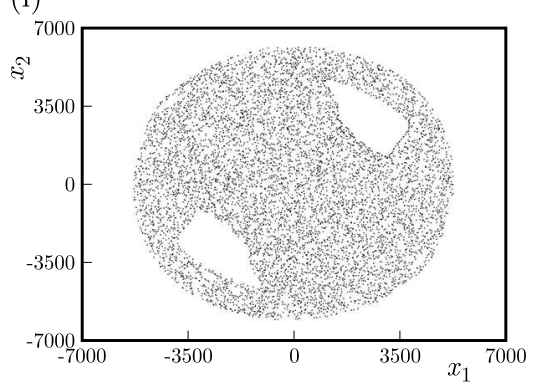

Fig. 6. The plate without damping - phase portraits (a), (d), (g), FFT analysis (b), (e), (h) and Poincaré maps (c), (f), (i) for the areas of dynamic stability (a), (b), (c), dynamic instability quasi-periodic solution (d), (e), (f) and dynamic instability - chaotic solution (g), (h), (i)

Applying FFT analysis, it can be concluded that it is possible to precisely determine dominant frequencies in the stability range (Fig. 6b). Also in the instability range with a quasi-periodic solution, dominant frequencies can be specified (Fig. 6e). In both cases, the appearance of two 
disproportionate to each other frequencies can be observed. The so-called two-dimensional torus (2D torus) is created. In both Fig. 6b and Fig. 6e, the largest Lyapunov exponents are approximately equal to zero $\left(\lambda_{1}=0.000002, \lambda_{2}=-0.000002\right.$ - for the point $k=0.50, \psi / 2 \Omega=0.30$; $\lambda_{1}=0.000004, \lambda_{2}=-0.000004-$ for the point $\left.k=0.25, \psi / 2 \Omega=1.00\right)$. It should be noted that the two zero Lyapunov exponents for the stability area are the result of the absence of damping in the system (2.3). As a consequence, there is no attractor (attractors) to which the trajectory would converge.

In the instability range with a chaotic solution (Fig. 6h), the frequency spectrum is continuous. It is not possible to specify the dominant frequencies. The amplitude of the tested signal increases significantly, which is expressed in decibels. The value of the largest Lyapunov exponent is positive $\left(\lambda_{1}=0.043531, \lambda_{2}=-0.043531\right)$.

Similarly to the plate without damping, the loss of stability for the plate with damping is associated with a sudden increase in the displacement $x_{1}$ and velocity $x_{2}$ (Figs. 7d, 7f, 7g, 7i) when compared to the dynamic stability areas (Fig. 7a). In the stability area - as a result of the introduced damping - the trajectory goes to the critical point (Fig. 7a). The Lyapunov exponents are negative $\left(\lambda_{1}=-0.019993, \lambda_{2}=-0.020007\right)$ and there is no solution in the FFT graphs (Fig. 7b) as well as Poincaré maps (Fig. 7c).

(a)

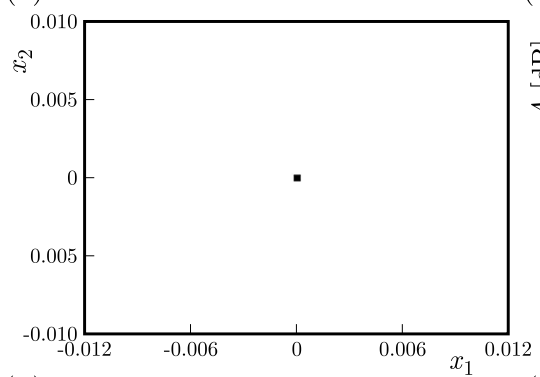

(d)

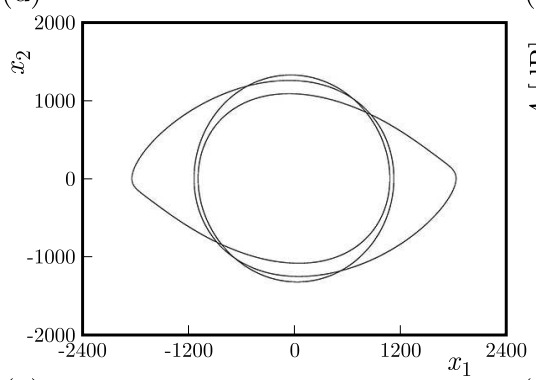

(g)

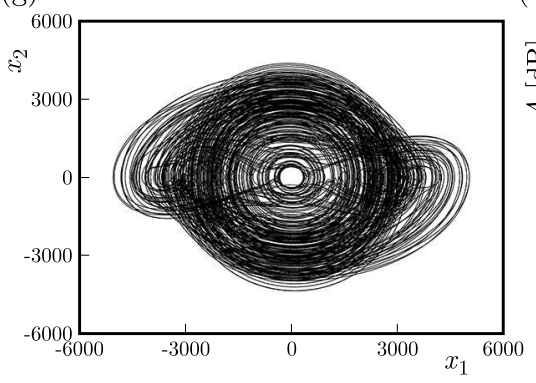

(b)

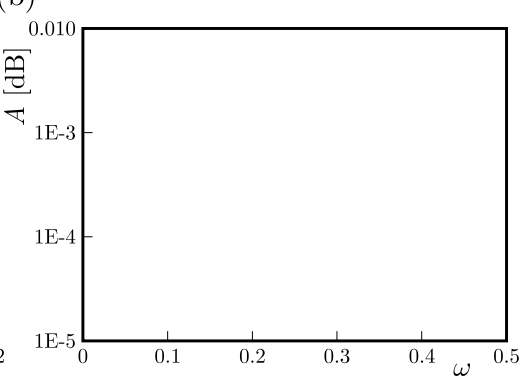

(e)

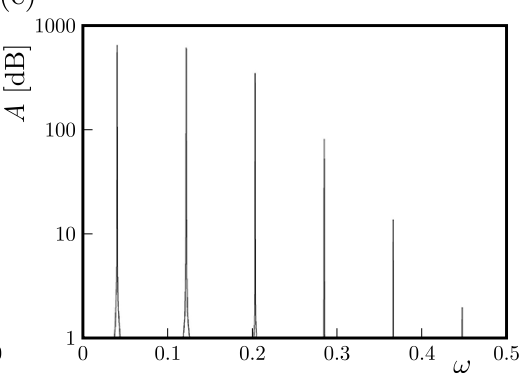

(h)

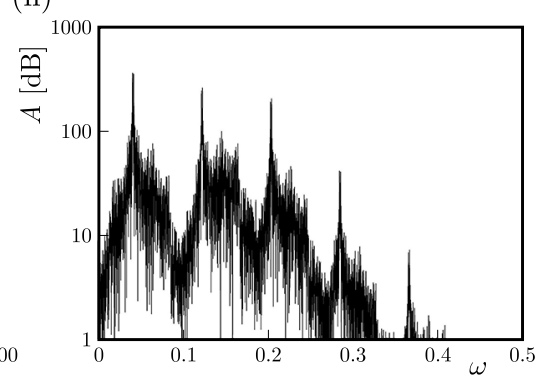

(c)

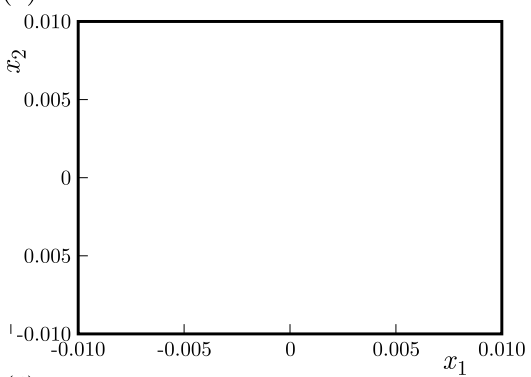

(f)

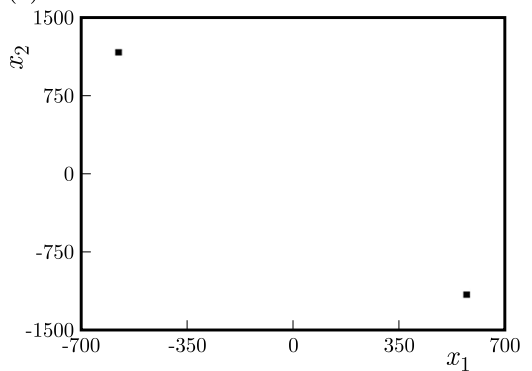

(i)

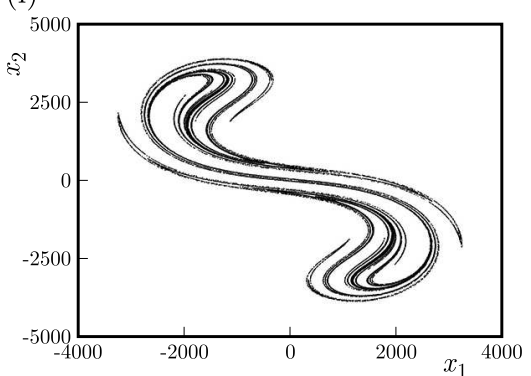

Fig. 7. The plate with damping - phase portraits (a), (d), (g), FFT analysis (b), (e), (h) and Poincaré maps (c), (f), (i) for the areas of dynamic stability (a), (b), (c), dynamic instability - periodic solution (d), (e), (f) and dynamic instability - chaotic solution (g), (h), (i)

In the areas of dynamic instability, a periodic solution has been obtained (Figs. 7d, 7e, 7f). Together with the series of period-doubling bifurcations, it leads to a chaotic solution (Figs. $7 \mathrm{~g}$, $7 \mathrm{~h}, 7 \mathrm{i}$ ). Figures $7 \mathrm{~d}, 7 \mathrm{e}, 7 \mathrm{f}$ show a solution with a period equal to 2 . The FFT analysis (Fig. 7e) 
enables precise representation of the dominant frequencies. The Lyapunov exponents are negative $\left(\lambda_{1}=-0.020000, \lambda_{2}=-0.020000\right)$.

Similarly to the plate without the damping effect, in the areas of dynamic instability with a chaotic solution, the frequency spectrum is continuous, and it is impossible to distinguish the dominant frequencies (Fig. $7 \mathrm{~h}$ ). The amplitude of the signal also increases. The value of the largest Lyapunov exponent is positive $\left(\lambda_{1}=0.043397, \lambda_{2}=-0.043397\right)$.

\section{Summary}

The subject of the research is to present the influence of the damping effect on the dynamic response for an isotropic plate. The areas of dynamic stability and instability for the plate with and without damping are compared. Additionally, using the criteria such as phase portraits, Poincaré maps, FFT analysis, the largest Lyapunov exponents, the nature of the solution of the analyzed plate has been presented.

After the tests, it can be concluded that the impact of damping causes changes in the instability areas of the studied structure. In addition, the introduction of damping to the system results in a significant difference in the occurrence of areas in which the solution is chaotic.

For the plate without damping, a quasi-periodic solution in the dynamic stability areas has been observed. The occurrence of two disproportionate to each other frequencies as well as formation of a $2 \mathrm{D}$ torus have been proved. Whereas, both the quasi-periodic as well as the chaotic solution in the instability range have been specified.

For the plate with the damping effect in the area of dynamic stability, the phase trajectory is going to the critical point. In the range of dynamic instability, the periodic solutions as well as the series of period-doubling bifurcations, which lead to the chaotic response, have been obtained.

In both analyzed cases (for the plate without and with damping), the loss of dynamic stability is associated with a significant increase in the displacement $x_{1}$ and velocity $x_{2}$ in comparison to the dynamic stability areas - the criteria of phase portraits and Poincaré maps. Using the FFT analysis, the loss of dynamic stability results in inability to precisely specify the dominant frequencies in the spectral signal (what is possible in the areas of dynamic stability), and a significant increase in their amplitude is found. Implementing the criterion of the largest Lyapunov exponents, it is possible to clearly present significant differences between the areas with a chaotic solution for plates without and with the damping effect.

Conflict of interest

The author declares that there is no conflict of interest concerning publication of this article.

\section{References}

1. Alijani F., Amabili M., Karagiozis K., Bakhtiari-Nejad F., 2011a, Nonlinear vibrations of functionally graded doubly curved shallow shells, Journal of Sound and Vibration, 330, 7, 1432-1454

2. Alijani F., Bakhtiari-Nejad F., Amabili M., 2011b, Nonlinear vibrations of FGM rectangular plates in thermal environments, Nonlinear Dynamics, 66, 3, 251-270

3. Ari-Gur J., Simonetta S.R., 1997, Dynamic pulse buckling of rectangular composite plates, Composites Part B: Engineering, 28, 3, 301-308

4. Bazant Z.P., Cedolin L., 2010, Stability of Structures: Elastic, Inelastic, Fracture and Damage Theories, World Scientific 
5. Bolotin V., 1962, Dynamic Stability of Elastic Systems, Vol. 1

6. Borkowski L., 2017, Numerical analysis of dynamic stability of an isotropic plate by applying tools used in dynamics, [In:] Dynamical Systems in Theoretical Perspective, J. Awrejcewicz (Edit.), Springer

7. Budiansky B., Roth R.S., 1962, Axisymmetric dynamic buckling of clamped shallow spherical shells, NASA TND1510, 597-606

8. Collatz L., 2012, The Numerical Treatment of Differential Equations, Springer Science and Business Media

9. Cooley J.W., Tukey J.W., 1965, An algorithm for the machine calculation of complex Fourier series, Mathematics of Computation, 19, 90, 297-301

10. Fortuna Z., Macukow B., Wasowski J., 2005, Metody numeryczne, WNT, Warszawa, ISBN 83-204-3075-5

11. Gilat R., Aboudi J., 2000, Parametric stability of non-linearly elastic composite plates by Lyapunov exponents, Journal of Sound and Vibration, 235, 4, 627-637

12. Hsu Y.C., Forman R.G., 1975, Elastic-plastic analysis of an infinite sheet having a circular hole under pressure, Journal of Applied Mechanics, 42, 2, 347-352

13. Hutchinson J.W., Budiansky B., 1966, Dynamic buckling estimates, AIAA Journal, 4, 3, $525-530$

14. Kleiber M., Kotula W., Saran M., 1987, Numerical analysis of dynamic quasi-bifurcation, Engineering Computations, 4, 1, 48-52

15. Kolakowski Z., 2007, Some aspects of dynamic interactive buckling of composite columns, Thin- Walled Structures, 45, 10, 866-871

16. Kolakowski Z., Kubiak T., 2007, Interactive dynamic buckling of orthotropic thin-walled channels subjected to in-plane pulse loading, Composite Structures, 81, 2, 222-232

17. Kolakowski Z., Teter A., 2013, Influence of inherent material damping on the dynamic buckling of composite columns with open cross-sections, Mechanics and Mechanical Engineering, 17, 1, 59-69

18. Kowal-Michalska K., 2010, About some important parameters in dynamic buckling analysis of plated structures subjected to pulse loading, Mechanics and Mechanical Engineering, 14, 2, 269-279

19. Kubiak T., 2007, Criteria of dynamic buckling estimation of thin-walled structures, Thin-Walled Structures, 45, 10, 888-892

20. Kubiak T., Kolakowski Z., Kowal-Michalska K., Mania R., Swiniarski J., 2010, Dynamic response of conical and spherical shell structures subjected to blast pressure, Proceedings of SSDS'Rio

21. Mania R., Kowal-Michalska K., 2007, Behaviour of composite columns of closed cross-section under in-plane compressive pulse loading, Thin-Walled Structures, 45, 10, 902-905

22. Michlin S.G., Smolnicki C.L., 1970, Approximate Methods for the Solution of Integral and Differential Equations, PWN, Warsaw

23. Moorthy J., Reddy J.N., Plaut R.H., 1990, Parametric instability of laminated composite plates with transverse shear deformation, International Journal of Solids and Structures, 26, 7, $801-811$

24. Petry D., Fahlbusch G., 2000, Dynamic buckling of thin isotropic plates subjected to in-plane impact, Thin-Walled Structures, 38, 3, 267-283

25. Raftoyiannis I.G., Kounadis A.N., 2000, Dynamic buckling of 2-DOF systems with mode interaction under step loading, International Journal of Non-Linear Mechanics, 35, 3, 531-542

26. Shariyat M., 2007, Thermal buckling analysis of rectangular composite plates with temperaturedependent properties based on a layerwise theory, Thin-Walled Structures, 45, 4, 439-452 
27. Tounti D., Cederbaum G., 1995, Influence of large deflections on the dynamic stability of nonlinear viscoelastic plates, Acta Mechanica, 113, 1-4, 215-231

28. Volmir A.S., 1972, Nonlinear Dynamics Plates and Shells, Moscow, Science

29. Wang Y.G., Song H.F., Li D., Wang J., 2010, Bifurcations and chaos in a periodic time-varying temperature-excited bimetallic shallow shell of revolution, Archive of Applied Mechanics, 80, 7, 815-828

30. Wu G.Y., Sнin Y.S., 2006, Analysis of dynamic instability for arbitrarily laminated skew plates, Journal of Sound and Vibration, 292, 1, 315-340

31. Yen Y.L., LAi H.Y., 2002, Chaotic and bifurcation dynamics for a simply supported rectangular plate of thermo-mechanical coupling in large deflection, Chaos, Solitons and Fractals, 13, 7, 1493-1506

32. Yuda H., Zhiqiang Z., 2011, Bifurcation and chaos of thin circular functionally graded plate in thermal environment, Chaos, Solitons and Fractals, 44, 9, 739-750

33. Zhang T., Liu T.G., Zhao Y., Luo J.Z., 2004, Nonlinear dynamic buckling of stiffened plates under in-plane impact load, Journal of Zhejiang University - Science A, 5, 5, 609-617

34. ZizicAs G.A., 1952, Dynamic buckling of thin plates, Transactions ASME, 74, 7, 1257-1268 